\title{
Viewing aortic dissection through a funnel?
}

\author{
Naveed U. Saqib, MD, and Anthony L. Estrera, MD
}

See related article on pages 108-15.

Development of malperfusion syndrome (MPS) remains a significant risk factor for morbidity and mortality in patients with acute aortic dissection, despite the progress and advancement in care resulting in overall improved outcomes for patients with acute aortic dissection of either type A or type B.

In their report in this issue of the Journal, Vendrell and colleagues ${ }^{1}$ highlight a novel variation of endovascular fenestration (funnel technique) in patients with acute aortic dissection (type A and type B) with dynamic MPS for whom thoracic endovascular aortic repair with an endograft could not be performed because of anatomic constraints. The fenestration was performed $4 \mathrm{~cm}$ proximal to the celiac artery and maintained with stent placement (after balloon angioplasty) from false lumen to true lumen. In their report of 28 cases, the early results are commendable, with 2 deaths $(7 \%)$ and 5 postoperative complications (4 minor and 1 major with limb ischemia). Concomitant stenting was needed for static compression in 13 of 28 patients. The midterm results at 2 years are equally notable, with only 2 patients $(9 \%)$ needing open repair for thoracic aortic enlargement and a single asymptomatic stent fracture being noted. As expected, most of the patients with MPS during the study period were treated with thoracic endovascular aortic repair (41/70).

It is important to understand that MPS is a spectrum, and when reporting outcomes a clear distinction should be made between malperfusion and malperfusion with ischemia and end-organ dysfunction. An acute type A dissection with malperfusion but without end-organ ischemia is associated with a significantly better outcome than malperfusion with end-organ dysfunction. In this study, 8 of 28 patients were reported to have severe ischemia (correlating with MPS with end-organ ischemia). All complications and both deaths reported occurred in this group of patients. One

From the Department of Cardiothoracic and Vascular Surgery, University of Texas Medical School at Houston, Memorial Hermann Heart \& Vascular Institute, Houston, Tex.

Disclosures: Authors have nothing to disclose with regard to commercial support.

Received for publication April 14, 2015; accepted for publication April 15, 2015; available ahead of print May 8, 2015.

Address for reprints: Anthony L. Estrera, MD, Department of Cardiothoracic and Vascular Surgery, The University of Texas Houston Medical School, 6400 Fannin St, Suite 2850, Houston, TX 77030 (E-mail: Anthony.L.Estrera@uth.tmc.edu).

J Thorac Cardiovasc Surg 2015;150:116-7

0022-5223/\$36.00

Copyright (c) 2015 by The American Association for Thoracic Surgery

http://dx.doi.org/10.1016/j.jtcvs.2015.04.035 could argue that the mortality of $25 \%$ $(2 / 8)$ in this subset correlates with the high mortality in previous studies associated with endovascular fenestrations performed for MPS with end-organ dysfunction.

In addition, the natural history of

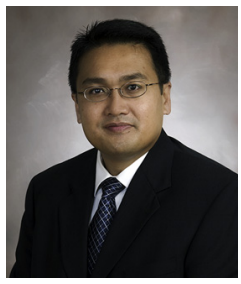
aortic dissection (with and without mal-

perfusion) is best outlined prognostically by differentiating between patients with involvement of the ascending aorta (type A aortic dissection) and patients with dissection confined to the distal arch and the descending aorta (type $\mathrm{B}$ dissection). This distinction is notable not only for the differing risk factors for development of dissection but also with regard to acute and chronic patient outcomes. We believe that it is important that the distinction be clearly made between malperfusion on presentation in patients with type $\mathrm{A}$ and type $\mathrm{B}$ dissection when presenting patient outcomes. Vendrell and colleagues ${ }^{1}$ reported that 19 of 28 patients had type A dissection and 9 of 28 had type B dissection; for early and long-term outcomes, however, both groups were reported together. Approximately one-third of patients with type A dissection manifest MPS on presentation. If the malperfusion is dynamic, restoration of true aortic luminal flow by eliminating the tear and replacing the ascending aorta will often correct the secondary malperfusion. Additional intervention for malperfusion thus may not be necessary.

Although Vendrell and colleagues ${ }^{1}$ are to be commended for their exceptional results, the study should be interpreted with prudence. The funnel technique with a stent from false lumen to true lumen appears to be an interesting and effective option for endovascular fenestration; however, because there was no randomized comparison between the previously established techniques of endovascular fenestrations, superiority of one technique relative to the other cannot be inferred. Additionally, with introduction of new branched thoracic endografts the anatomic suitability for thoracic endovascular aortic repair will likely increase, and the need for performing endovascular fenestration thus will likely decrease. Subsequent thoracic endovascular aortic repair after the funnel technique might be limited, necessitating open repair in patients with previous funnel technique in whom thoracic aortic aneurysmal degeneration occurs. Longer follow-up to determine safety and effect on subsequent repair is therefore needed. Although no aortic wall perforation or stent migration was seen in this cohort, serious complications can occur. In the end, the funnel technique is intriguing, but, as we all recognize, acute aortic 
dissection is very complex in its presentation as well as its management and requires one's eyes to be wide open, avoiding any "funnel vision" during treatment.

\section{Reference}

1. Vendrell A, Frandon J, Rodiere M, Chavanon O, Baguet JP, Bricault I, et al. Aortic dissection with acute malperfusion syndrome: endovascular fenestration by the funnel technique. J Thorac Cardiovasc Surg. 2015;150:108-15. 